\title{
Whether Delirium Acts as ECT?
}

Shailendra Mohan Tripathi, Rakesh Kumar Tripathi*, Indrapal Singh, Shrikant Srivastava and Tiwari SC

Department of Geriatric Mental Health, King George's Medical University (KGMU), India

\begin{abstract}
Delirium is defined by the acute onset of fluctuating cognitive impairment and a disturbance of consciousness with reduced ability to attend. It may occur at any age but is more common in older adults. By virtue of working in the psychogeriatric hospital (Department of Geriatric Mental Health, King George's Medical University, Lucknow, India), authors experienced that many elderly patients with psychiatric illness develops delirium because of various reasons. Subsequently, we observed that psychiatric manifestations of the patients improved significantly following recovery from delirium. A series of such four cases (case 1- Depression with psychotic features, 2- Manic episode, 3- Bipolar affective disorder current episode mania and 4- Depressive episode) later developed delirium has been presented and discussed in this paper. ICD-10 criteria were used for the diagnosis of delirium and psychiatric illnesses. Average stay of these patients in the hospital was 10 days. We found that signs and symptoms of these psychiatric disorders disappeared almost completely following the recovery from delirium. Therefore, it can be assumed that delirium potentially acts similar to ECT. By reporting such case we open a new dimension of research for effective treatment of the psychiatric patients. Inducing delirium in a patient is unethical but understanding pathophysiology behind it will certainly open the door for better cure of the ailing psychiatric patients.
\end{abstract}

Keywords: Delirium; Older adults; ECT

\section{Introduction}

Delirium is defined by the acute onset of fluctuating cognitive impairment and a disturbance of consciousness with reduced ability to attend. According to ICD-10 Delirium is an etiologically nonspecific syndrome characterized by concurrent disturbances of consciousness and attention, perception, thinking, memory, psychomotor behaviour, emotion, and the sleep-wake cycle. It may occur at any age but is most common after the age of 60 years [1]. None of the age is immune to the delirium, it can occur in anyone at any age but it is more common in older adults. In a review, it is reported that $15 \%$ to $30 \%$ of elderly patients will have delirium on admission to hospital and up to $56 \%$ will develop delirium during their stay [2]. The point prevalence of delirium in the community is $1.1 \%$ amongst the general population aged over 55 years and up to $14 \%$ in those over 85 years [3]. Since delirium is an acute medical condition, its prevalence can only be studied in hospital settings. A meta-analysis of 42 studies found delirium to be prevalent on admission in $10-31 \%$ of medical inpatients, and to occur in the hospital in 3-29\% [4]. Fong et al. [5] reported that the overall prevalence of delirium in the community is just $1-2 \%$, but in the setting of general hospital admission this increases to $14-24 \%$. The incidence of delirium arising during a hospital stay ranges from $6 \%$ to as high as $56 \%$, and this incidence is even higher when more-specialized populations are considered, including those in postoperative, intensive-care, subacute and palliative-care settings $[4,6,7]$. Postoperative delirium occurs in $15-53 \%$ of surgical patients over the age of 65 years [8] and among elderly patients admitted to an Intensive Care Unit (ICU) the delirium incidence can reach 70-87\% [9]. Advanced age and cognitive impairment are major risk factors for the development of delirium. About $15 \%$ of elderly inpatients in acute medical-surgical services have delirium at any time, and the rate is even higher among nursing home patients. Approximately 30 to 40 percent of hospitalized patients older than age 65 have an episode of delirium, and another 10 to 15 percent of elderly persons exhibit delirium on admission to the hospital $[10,11]$.

The patient with delirium presents with a wide range of psychiatric symptoms making it difficult to distinguish with other psychiatric disorders such as schizophrenia. Thought content disturbances of delirium entails themes from the immediate environment and circumstances, hallucinations used to be frequently visual rather than auditory and formal thought disorder typically comprised poverty of thinking and illogicality [12]. Clouding of consciousness and inattention found in delirium may be helpful in differentiating it from psychosis. The major causes of delirium are neurological disorders, systemic disease, metabolic disorders and either intoxication or withdrawal from pharmacological or toxic agents. Cause of delirium can also be multifactorial. The permeability of blood brain barrier is impaired by ageing and dementia, which may predispose the patient to delirium. The major neurotransmitter hypothesized to be involved in delirium is acetylcholine, which is decreased in the brain. One of the most common causes of delirium is toxicity from too many prescribed medications with anticholinergic activity. Noradrenaline, serotonin and glutamate have also been implicated in the causation of delirium. Neurotransmitters involve for the causation of the psychiatric disorders are same which are involved in the delirium [13-15]. It was observed that psycho-geriatric patients are at receiving end of development of delirium because of multiple drugs prescribed for the co-morbidities along with psychotropic medications. Development of delirium during course of treatment is frequent in psychogeriatric patients as compared to their younger counterparts. During course of treatment of the psychiatric patients who developed delirium in our hospital, we observed an interesting phenomenon. These patients had a psychiatric illness before the onset of delirium as per the ICD-10 criteria. Somehow after recovery from the delirium most of the psychiatric symptoms were disappeared. Written informed consent was taken from the patients and their family members to present and publish the case for the benefit of the scientific community. Their names are written as Mr. A, B, C and D to maintain confidentiality. A series of 4 cases is being presented below and discussed.

*Corresponding author: Rakesh Kumar Tripathi, Department of Geriatric Mental Health, King George's Medical University (KGMU), India, E-mail: rastripathi@yahoo.com

Received March 18, 2013; Accepted July 15, 2013; Published July 24, 2013

Citation: Tripathi SM, Tripathi RK, Singh I, Srivastava S, Tiwari SC (2013) Whether Delirium Acts as ECT? Aging Sci 1: 107. doi: 10.4172/2329-8847.1000107

Copyright: @ 2013 Tripathi SM, et al. This is an open-access article distributed under the terms of the Creative Commons Attribution License, which permits unrestricted use, distribution, and reproduction in any medium, provided the original author and source are credited. 


\section{Case 1}

Mr. A, 61 year old male presented to the Department of Geriatric Mental Health, (DGMH) OPD with chief complaints of low mood, restlessness, decreased interaction, decreased appetite, decreased sleep and suspiciousness for the last 1 month. On mental status examination he was depressed. He was having persecutory delusions and auditory hallucinations. On enquiry he complaints that the voices are coming from the outside, they abuse him and threatened to kill him. The provisional diagnosis of depression with psychotic features was entertained and was prescribed escitalopram $10 \mathrm{mg}$, olanzapine $5 \mathrm{mg}$ and lorazepam $2 \mathrm{mg}$ per day. His routine blood investigations and CT Head were within normal limits. On follow-up after 15 days of treatment he was confused. He developed visual hallucinations that insects are crawling on the ground. His sleep cycle was reversed. Possibility of drug induced delirium was kept. His CBC, electrolytes, blood urea, serum creatinine and CT scan Head were within normal limits. Delirium was managed by withdrawing all the psychotropic medications prescribing multivitamins and electrol powder. On followup next week Mr. A, recovered from the delirium. Interestingly his all psychiatric symptoms were improved. Family members reported him as normal as he was earlier. On follow-up after three weeks, there was re-emergence of the symptoms but of very low intensity. So he was prescribed escitalopram $10 \mathrm{mg}$ and olanzapine $5 \mathrm{mg}$ and he has been maintaining well on this treatment.

\section{Case 2}

Mr. B, 64 year old retired male presented to the DGMH OPD with chief complaints of over talkativeness, aggressiveness, doing big claims and decreased need for the sleep for the last 1 month. He often suspicious about the son that he had taken out money from his account for which he used to become agitated. On mental status examination he was elated, having delusions of grandiosity and theft. On detailed evaluation it was his suspiciousness about the money being withdrawn from his account has started little earlier than the overtalkativeness. Provisional diagnosis of $1^{\text {st }}$ episode mania was made. He was hospitalised and he was given haloperidol and promethazine in the form of injectibles. He was not accepting medicines and food orally so the injectibles were continued further for the 2 more days but on $3^{\text {rd }}$ day of hospitalization he developed confusion. He was not oriented to time place and person. His sleep cycle was reversed and used to pick something from his belongings. Possibility of drug induced delirium was kept and injectibles were stopped. His all blood parameters were within normal limits. No abnormality detected on CT scan head except cerebral atrophy. He was kept on the Multivitamins and electrol powder. Hydration was maintained. Finally he recovered from the delirium but interestingly all his symptoms for which he was hospitalized were gone. He was in the state of remission and was discharged. Later on followup 1 month after discharge he developed forgetfulness and delusion of theft and on subsequent visits he was diagnosed as a case of Dementia of Alzheimer's type. Rivastigmine $3 \mathrm{mg}$ per day in divided doses was started and hiked up to $9 \mathrm{mg}$. Now he has been maintaining well on the rivastigmine.

\section{Case 3}

Mr. C, 63 year old male presented to DGMH OPD with chief complaints of overtalkativeness, aggressiveness, irritability, blaming his family members about the mistreatment for last 15 days. On mental state examination his psychomotor activity was increased and mood was irritable. He had similar episode 2 years back which got treated from the DGMH and completely recovered within 2 months and discontinued the treatment without consultation. Provisional diagnosis of Bipolar Affective Disorder current episode Mania was entertained. $\mathrm{He}$ was hospitalized. Combination of oral haloperidol $15 \mathrm{mg}$ and combination of chlorpromazine $(200 \mathrm{mg})$ and trihexiphenidyl $8 \mathrm{mg}$ was started but instead of improving, his symptoms started worsening after initial glimpse of improvement. He was quite agitated, trying to jump from the bed, was not oriented to time, place and person.He often visualizes small creatures creeping on the ground. Diagnosis of drug induced delirium was made and all the drugs were stopped and promethazine $25 \mathrm{mg}$ once daily at night, electrol powder and multivitamins were prescribed to him. CT scan Head and routine blood investigations were within normal limits. He recovered from the delirium but interestingly he reached into remission. Later on he was discharged on Mood stabilizer (sodium valproate) to prevent further relapse.

\section{Case 4}

Mr. D, 66 year old male presented to DGMH OPD with chief complaints of not recognizing family members, not eating, not sleeping in the night. He used to take naps during day hours. He was hospitalized. Provisional diagnosis of delirium was made. On further evaluation it was found that patient was sick for the more than a year. He was less communicative, feels low most of the time and often complaints of heaviness in head. The symptoms worsen early in the morning. He also had disturbed sleep especially had early morning awakening. For which he consulted many private practitioners without much of the effect in his clinical condition. On investigations his all blood parameters and CT scan Head were within normal limits except serum sodium. Serum sodium was $124 \mathrm{meq} / \mathrm{l}$. He was given normal saline as well as oral salt to correct hyponatremia. The hyponatremia was thought be related to the poor oral intake secondary to depression he had. Hyponatremia lead him to delirium. On recovery from delirium family members reported that patient's past and present complaints were completely disappeared. He was discharged without medication and on follow-up he has been maintaining well.

\section{Discussion}

It is well proven that a marked increase in slow wave activity in interictal period on EEG may last for weeks to months after completion of an ECT-course [16,17]. ECT cumulatively leads to Electroencephalogram (EEG) changes characterized by increased amplitude and reduced frequency ('delta' activity) somewhat similar to delirium [18]. It is likely that such EEG changes are correlated with improved clinical response rates and reduced likelihood of relapse [19]. EEG characteristics of delirium include slowing or dropout of the posterior dominant rhythm, generalized theta or delta slow-wave activity, poor organization of the background rhythm, and loss of reactivity of the EEG to eye opening and closing [20]. It might be possible on the above theory that ultimate effect of ECT is not because of seizure activity but because of the postictal confusion which causes delirious state in the patient. It means that if by any mean psychiatric patient reach to delirious state the psychiatric symptoms may be subsided. Delirium is a common and serious acute neuropsychiatric syndrome with core features of inattention and cognitive impairment, and associated features including changes in arousal, altered sleep-wake cycle, and other changes in mental status [21]. Hypotheses about the pathophysiology of delirium are speculative and largely based on animal research. According to the neurotransmitter hypothesis, decreased oxidative metabolism in the brain causes cerebral dysfunction due to abnormalities of various neurotransmitter systems. Reduced cholinergic function, excess release of dopamine, 
Citation: Tripathi SM, Tripathi RK, Singh I, Srivastava S, Tiwari SC (2013) Whether Delirium Acts as ECT? Aging Sci 1: 107. doi: 10.4172/23298847.1000107

norepinephrine, and glutamate, and both decreased and increased serotonergic and gamma-aminobutyric acid activity may underlie the different symptoms and clinical presentations of delirium. Furthermore, severe illness and physiologic stress may give rise to modification of blood-brain barrier permeability and increased activity of the hypothalamic-pituitary-adrenal axis. These circumstances possibly also contribute to changes in neurotransmitter synthesis and release of cytokines in the brain, and consequently to the occurrence of delirium. Elderly patients are more at risk for developing delirium, very likely due to age-related cerebral changes in stress-regulating neurotransmitter and intracellular signal transduction systems [14]. Psychiatric disorders are result of disturbances in above mentioned neurotransmitters either increased or decreased. If neurotransmitter system is disturbed possibly they get rearranged on recovery and ultimately result in remission. Three of the four patients developed drug induced delirium and on recovery the psychiatric symptoms were completely absent. One of the four patients hospitalized for delirium with background history suggestive of depression. On recovery from the delirium there were no symptoms suggestive of depression. One of the four patients relapsed again on follow-up suggestive that recovery is not lifelong and it has to be relapsed after few days if not given psychotropic medication as we see in the cases of ECT. On the basis of above discussion we can say that delirium acts as ECT. If a less invasive substitute for ECT is desired, the priority would be to explore underlying mechanism of delirium which has similar effect on psychiatric disorders. By reporting such cases we open a new dimension of research by virtue of which we can treat effectively the psychiatric patients. Inducing delirium in a patient is unethical but understanding pathophysiology behind it will certainly open the door for better cure of the ailing psychiatric patients.

\section{References}

1. World Health Organization (WHO) (1993) The ICD-10 classification of mental and behavioural disorders; diagnostic criteria for research.

2. Wass S, Webster PJ, Nair BR (2008) Delirium in the elderly: A Review. Oman Med J 23: 150-157

3. Rahkonen T, Eloniemi-Sulkava U, Paanila S, Halonen P, Sivenius J, et al (2001) Systematic intervention for supporting community care of elderly people after a delirium episode. Int Psychogeriatr 13: 37-49.

4. Siddiqi N, House AO, Holmes JD (2006) Occurrence and outcome of delirium in medical in-patients: a systematic literature review. Age Ageing 35: 350-364.
5. Fong TG, Tulebaev SR, Inouye SK (2009) Delirium in elderly adults: diagnosis, prevention and treatment. Nat Rev Neurol 5: 210-220.

6. Bruce AJ, Ritchie CW, Blizard R, Lai R, Raven P (2007) The incidence of delirium associated with orthopedic surgery: a meta-analytic review. Int Psychogeriatr 19: 197-214.

7. Girard TD, Ely EW (2008) Delirium in the critically ill patient. Handb Clin Neurol 90: 39-56.

8. Inouye SK (2006) Delirium in older persons. N Engl J Med 354: 1157-1165.

9. Pisani MA, McNicoll L, Inouye SK (2003) Cognitive impairment in the intensive care unit. Clin Chest Med 24: 727-737.

10. Horacek R, Prasko J, Mainerova B, Lstalova K, Grosmanova T et al. (2011) Delirium in surgery intensive care unit. Act Nerv Super Rediviva 53: 121-132.

11. Maldonado JR (2008) Delirium in the Acute Care Setting: Characteristics Diagnosis and Treatment. Crit Care Clin 24: 657-722.

12. Cutting $\mathrm{J}(1987)$ The phenomenology of acute organic psychosis. Comparison with acute schizophrenia. Br J Psychiatry 151: 324-332.

13. Maldonado JR (2008) Pathoetiological model of delirium: a comprehensive understanding of the neurobiology of delirium and an evidence-based approach to prevention and treatment. Crit Care Clin 24: 789-856.

14. Vander Mast RC (1998) Pathophysiology of delirium. J Geriatr Psychiatry Neurol 11: 138-145.

15. Hshieh TT, Fong TG, Marcantonio ER, Inouye SK (2008) Cholinergic deficiency hypothesis in delirium: a synthesis of current evidence. J Gerontol A Biol Sci Med Sci 63: 764-772.

16. Weiner RD, Rogers HJ, Davidson JR, Kahn EM (1986) Effects of electroconvulsive therapy upon brain electrical activity. Ann N Y Acad Sci 462: 270-281.

17. Sackeim HA, Luber B, Katzman GP, Moeller JR, Prudic J, et al. (1996) The effects of electroconvulsive therapy on quantitative electroencephalograms. Relationship to clinical outcome. Arch Gen Psychiatry 53: 814-824.

18. Niedermeyer E, da Silva L (1993) Electroencephalography: basic principles clinical applications, and related fields. (5thedn) Baltimore: Williams and Williams, USA.

19. Abrams R (1997) Electroconvulsive therapy 3rd (Edn.) Oxford University Press, USA.

20. Jacobson S, Jerrier H (2000) EEG in delirium. Semin Clin Neuropsychiatry 5 86-92.

21. Maclullich AM, Ferguson KJ, Miller T, de Rooij SE, Cunningham C (2008) Unravelling the pathophysiology of delirium: a focus on the role of aberrant stress responses. J Psychosom Res 65: 229-238. 